Article

\title{
Physiological Changes in Barley mlo-11 Powdery Mildew Resistance Conditioned by Tandem Repeat Copy Number
}

\author{
Cynthia Ge ${ }^{1}$, Paula Moolhuijzen ${ }^{1}{ }^{\mathbb{D}}$, Lee Hickey ${ }^{2}{ }^{\circledR}$, Elzette Wentzel ${ }^{1}$, Weiwei Deng ${ }^{1}$, \\ Eric G. Dinglasan ${ }^{2}\left(\mathbb{D}\right.$ and Simon R. Ellwood ${ }^{1, *(D)}$ \\ 1 Centre for Crop and Disease Management, School of Molecular and Life Sciences, Curtin University, \\ Bentley, WA 6102, Australia; cynthia.ge@curtin.edu.au (C.G.); paula.moolhuijzen@curtin.edu.au (P.M.); \\ Elzette.Wentzel@curtin.edu.au (E.W.); weiwei.deng@curtin.edu.au (W.D.) \\ 2 Centre Queensland Alliance for Agriculture and Food Innovation, The University of Queensland, \\ St. Lucia, QLD 4069, Australia; 1.hickey@uq.edu.au (L.H.); e.dinglasan@uq.edu.au (E.G.D.) \\ * Correspondence: simon.ellwood@curtin.edu.au
}

Received: 28 October 2020; Accepted: 17 November 2020; Published: 20 November 2020

\begin{abstract}
Wild barley accessions have evolved broad-spectrum defence against barley powdery mildew through recessive mlo mutations. However, the mlo defence response is associated with deleterious phenotypes with a cost to yield and fertility, with implications for natural fitness and agricultural productivity. This research elucidates the mechanism behind a novel $m l o$ allele, designated mlo-11(cnv2), which has a milder phenotype compared to standard mlo-11. Bisulphite sequencing and histone ChIP-seq analyses using near-isogenic lines showed pronounced repression of the Mlo promoter in standard mlo-11 compared to mlo-11(cnv2), with repression governed by $24 \mathrm{nt}$ heterochromatic small interfering RNAs. The mlo-11(cnv2) allele appears to largely reduce the physiological effects of mlo while still endorsing a high level of powdery mildew resistance. RNA sequencing showed that this is achieved through only partly restricted expression of $M l o$, allowing adequate temporal induction of defence genes during infection and expression close to wild-type Mlo levels in the absence of infection. The two mlo-11 alleles showed copy number proportionate oxidase and peroxidase expression levels during infection, but lower amino acid and aromatic compound biosynthesis compared to the null allele mlo-5. Examination of highly expressed genes revealed a common WRKY W-box binding motif (consensus ACCCGGGACTAAAGG) and a transcription factor more highly expressed in $m l o-11$ resistance. In conclusion, mlo-11(cnv2) appears to significantly mitigate the trade-off between $m l o$ defence and normal gene expression.
\end{abstract}

Keywords: Hordeum vulgare; Blumeria graminis f. sp. hordei; gene silencing; histone modification; reactive oxygen species (ROS)

\section{Introduction}

Barley is an important cereal crop globally, ranked fourth in terms of total quantity produced (http://www.fao.org/in-action/inpho/crop-compendium/cereals-grains/en/). Powdery mildews are obligate biotrophic fungal diseases which in barley are caused by Blumeria graminis f. sp. hordei (Bgh). The fungus stunts barley growth, reduces yield, and can downgrade grain quality from malting to animal feed. Broad-spectrum resistance to powdery mildew that confers resistance to all races of $B g h$ is determined by recessive mutations of the Mlo gene. Mlo is an enigmatic and broadly conserved plant gene with mlo-governed resistance reported in both monocot and dicot species (reviewed in [1]). In barley, over 30 artificially induced mlo alleles are known to exist [2]. The gene is responsive to 
biotic and abiotic stimuli, is involved in developmental processes, and is a negative regulator of cell death [3,4]. mlo alleles act via rapid formation of large cell-wall appositions (CWA) and necrosis that correlate with resistance in epidermal cells. The CWA phenotype is also observed in nonhost resistance to powdery mildew, for example, following inoculation of barley with wheat powdery mildew [5].

A natural form of barley mlo resistance, known as mlo-11, involves a wild-type Mlo gene that is silenced via an upstream tandem repeat array consisting of the Mlo $5^{\prime}$ UTR and exons 1-5 [6]. Both induced mutants and the original or standard mlo-11 exhibit undesirable side effects, with necrotic leaf spotting, chlorosis, and leaf senescence (Figure 1). Breeders have overcome these effects, with most European spring cultivars now containing mlo resistance [7,8]. Ge et al. [9] uncovered a second natural variant of mlo-11 with an apparently fewer pleiotropic effects, named mlo-11(cnv2), which showed partial resistance to powdery mildew in seedling leaves and full resistance in adult leaves. The variant had just two copies of the tandem repeat array, compared to 11-12 in standard mlo-11, and lacked spontaneous cell death in uninfected leaves and death of underlying mesophyll cells during infection, characteristic of $m l o$ alleles with a stronger effect. Other partial mlo resistance phenotypes, conferred by missense mutations, have been reported in barley (mlo-12 and mlo-28 [3]) and a series of alleles provide varying levels of potency in wheat [10].

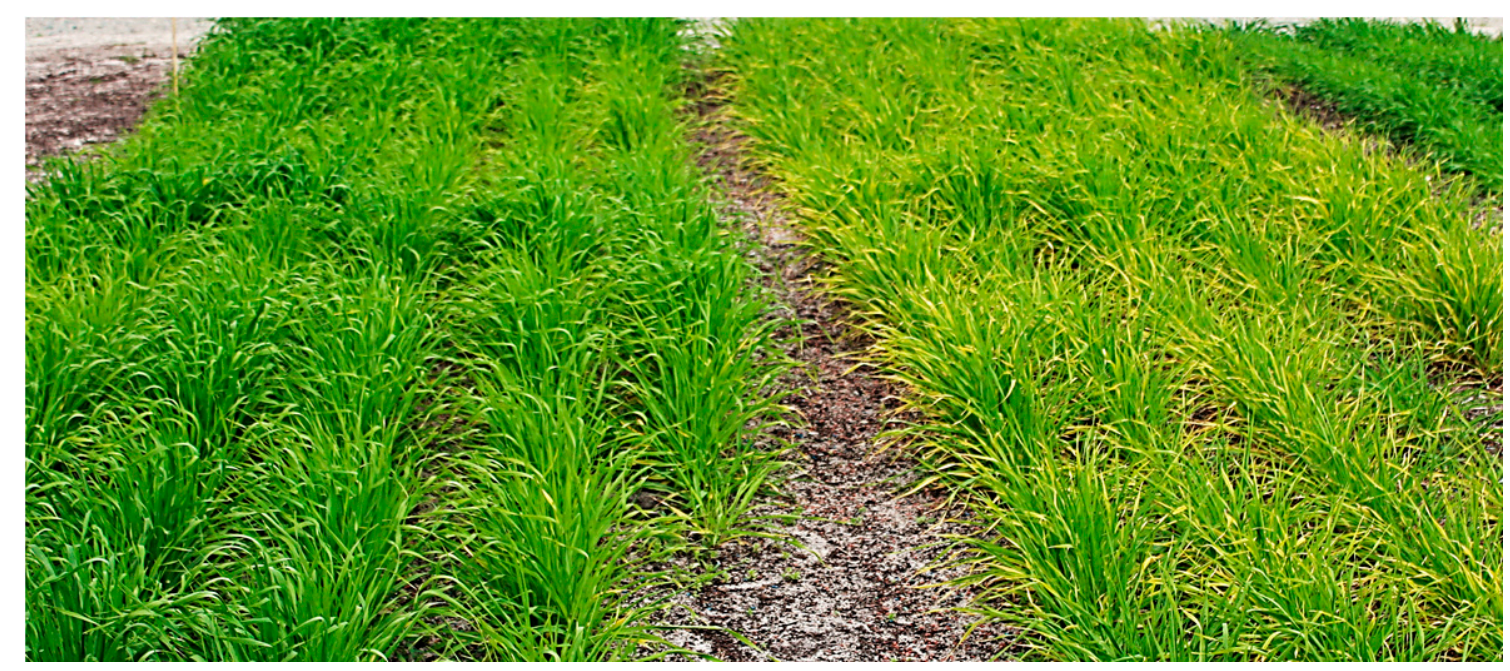

Figure 1. Field-grown barley Pallas $22(m l o-5)$ plants displaying chlorosis and senescence on the right-hand side, compared to a wild-type Mlo line Pallas 18 on the left-hand side.

The $m l o-11$ (cnv2) phenotype appears to correspond to the relative Mlo expression level differences compared to standard mlo-11 [9], and, on the basis of DNA methylation sequencing, the authors postulated that this may involve small RNA-directed DNA methylation (RdDM). DNA methylation was first discovered in plants, and research has consequently demonstrated an elaborate role in plant development and environmental responses through histone modifications [11,12]. Previous studies revealed silencing of transposable elements and repetitive heterochromatic sequences by RdDM [13] and histone H3K9 methylation [14], while repression of regions and genes in euchromatic regions occurs via H3K27 methylation [15]. H3K4 methylation, associated with actively transcribed genes, occurs in euchromatic regions. In addition to methylation, acetylation of histones leads to remodelling of condensed chromatin, leading to gene activation [16].

The precise functions and molecular basis of mlo resistance remain little understood, although a role in immunity or suppression of defence responses is implicit with the wild-type gene negatively regulating plant defence responses, with similar sets of co-expressed genes demonstrated in both Arabidopsis and barley $[4,17,18]$. Aside from measurable yield and phenotypic effects associated with mlo alleles, resource requirements of this resistance and defence against pathogens in general have been a matter of some conjecture. A trade-off between energy for growth and activation of defence 
responses was considered to be a question of balancing finite resources and the interactions between different signalling pathways [19]. More recent findings suggest that an antagonistic relationship between growth and defence results from incompatible molecular pathways or sharing of signalling components. One such example is the target genes for the HBI1 transcription factor (TF) which differentially regulates incompatible reactive oxygen species (ROS) requirements, controlling both cell growth and resistance to Pseudomonas syringae [20].

Phenotypic differences between the two mlo-11 variants provided a hypothesis that they act in measurably different growth or defence modes irrespective of the presence or absence of pathogens. With this in mind, we generated near-isogenic lines (NILs) where the resistance domains were introgressed into the barley powdery mildew-susceptible cultivar (cv) Baudin. For comparison, two Pallas NILs [21] with and without the mlo-5 null mutation were included. The principal objectives of this study were to examine the mechanisms involved in epigenetic gene regulation and to explore global gene expression pathways, common regulatory elements, and resource allocation differences between the two mlo-11 variants that may explain the phenotypic differences.

\section{Results}

Near-isogenic lines containing standard mlo-11 and mlo-11(cnv2) were created. This removes different donor parent genetic backgrounds that may affect phenotypic expression. To confirm $m l o-11$ subunit number stability during multiple meiotic events, digital PCR (APCR) indicated that, excluding one copy of Mlo from the wild-type Mlo gene, the mlo-11 subunit repeat numbers were 12 for standard $m l o-11$, two for mlo-11(cnv2), and zero in cv Baudin (wild-type Mlo), abbreviated as S12, S2 and S0, respectively. dPCR subunit copy number averages were 12.213 (standard error $(\mathrm{SE})=0.114, p<0.001$ ), $2.162(\mathrm{SE}=0.121, p<0.001)$, and $1.123(\mathrm{SE}=0.026, p<0.001)$ for $\mathrm{S} 12, \mathrm{~S} 2$, and $\mathrm{S} 0$, respectively.

\subsection{Near-Isogenic Line Macroscopic and Cytological Analysis}

Comparative macroscopic and cytological analyses between $m l o-11$ variants and wild-type $M l o$ were conducted to establish variant phenotypes in the new NILs. For macroscopic phenotypes, the firstand third-leaf stages were selected to compare the number and size of powdery mildew colonies between the Baudin NILs. The biological significance of these stages lies in the first-leaf stage being generally being more susceptible in mlo lines than older leaves, represented by the third leaf. These leaf stages allow differences in onset of mlo resistance to be characterised before the emergence of fully expanded fifth leaves, which are coincident with full resistance in milder alleles such as mlo-11(cnv2) [9]. Detached leaf colony counts showed fewer colonies developed on S2 at the first-leaf stage than on S0 (Figure 2A), while, at the third-leaf stage, colonies progressed more slowly than in S0, with an infection type (IT) 2-3 rather than 4 at 14 days post inoculation (dpi) (Figure 2B). On the third expanded leaves, both S2 and S12 showed much smaller colonies than S0, which had a significantly larger number of colonies than S12 (Figure 2C). On the first expanded leaves, S0 and S2 colony sizes were of similar sizes, whereas S12 were much smaller (Figure 2D). Leaves from the S2 and S12 at the fifth-leaf stage showed no colony formation (data not shown).

In terms of whole-adult-plant phenotypic differences between the NILs, the most pronounced were for the point mutation mlo-5 in the Pallas NIL P22 and the control P18. P22 plants grown in outdoor plots showed leaf senescence and physiological spotting compared to their P18 counterparts (Figure 1). At the cytological level, the mlo resistance genes in this study shared two prominent features, cell-wall appositions (CWAs or papillae) and mesophyll cell death. Cytological differences occurred in the size and staining intensity of CWA at attempted Bgh penetration sites. P22 (mlo-5) had the largest and most intensely stained CWA (Figure S1, Supplementary Materials), followed by S12 and S2 (Figure 3A-F). Despite three staining methods being used, (double staining with aniline blue and Evans blue (Figure 3A,B), trypan blue (Figure 3B,C), and 3,3'-diaminobenzidine (DAB) (Figure 3E,F), CWAs were the predominant feature shared by S2 and S12. In the absence of Bgh inoculation, S2 lacked spontaneous CWAs or extensive mesophyll cell death (Figure 3H,J), which was present in S12 (Figure 3I) 
and P22 (not shown), with mesophyll cell death only occasionally observed and limited to a few cells (Figure 3I). Cytosolic vesicles were not obvious in the mlo lines at $72 \mathrm{~h}$ post infection (hpi). These are considered as multifunctional defence agents and are known to accrue around CWAs at attempted penetration sites by biotrophs $[22,23]$. We also compared these symptoms with infection by barley by wheat mildew, B. graminis f. sp. tritici $(B g t)$, where the nonhost interaction at the site of attempted penetration showed weakly defined CWA; however, a large DAB stain halo was evident. CWAs and pigmentation at the penetration site are a feature of biotrophic nonhost interactions [5]. Alongside host cytological responses, the pathogen also displayed different characteristics in different Baudin NIL lines. Bgh germinating conidia showed the highest ratio of secondary appressorium tubes (SAT) and secondary primary tubes (PGT) in P22 (Figure 3K,L) and S12 (Figure S1, Supplementary Materials), following unsuccessful primary penetration events.

A

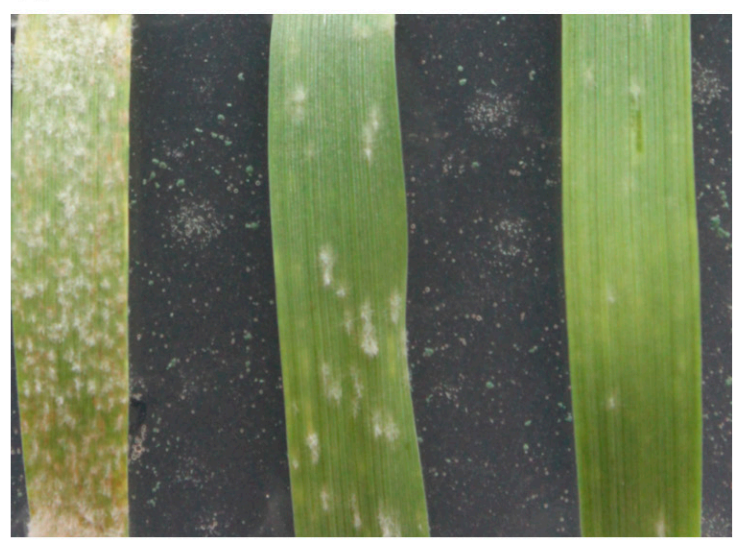

\section{C}

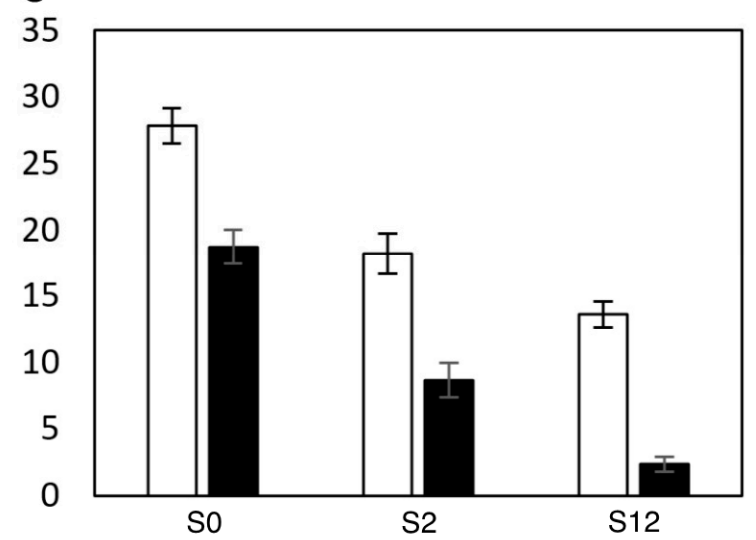

$\square$ 1st leaf colonies
B

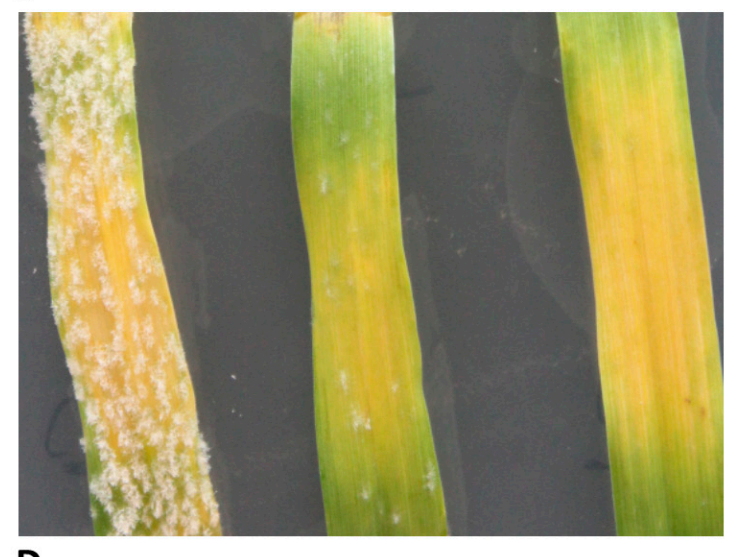

D

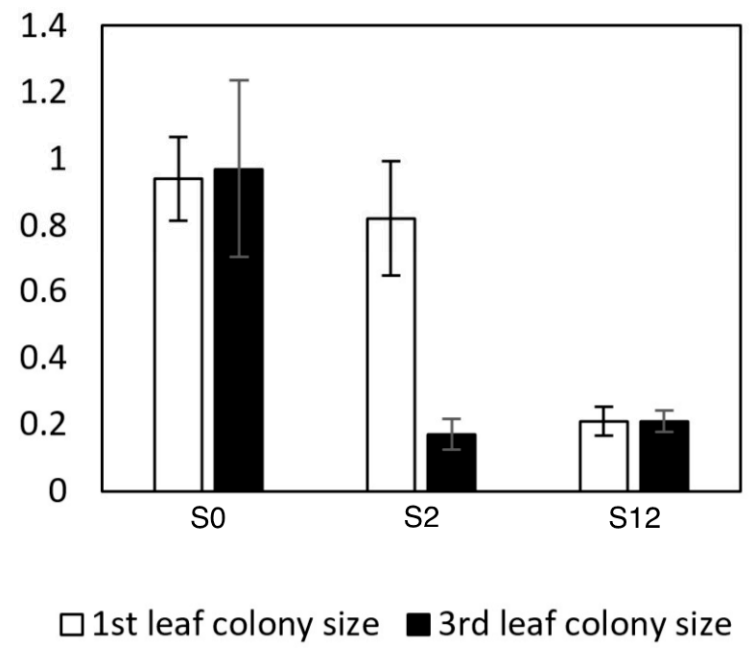

Figure 2. Colony number and size differences in detached leaf assays for the first (A) and third leaves (B) with isolate Chi-001. Leaves are ordered from left to right by S0 (Mlo wild-type control), S2 (mlo-11(cnv2), and S12 (mlo-11), respectively. Colony counts per $\mathrm{cm}^{2}$ and sizes in $\mathrm{mm}$ are shown in (C) and (D), respectively, according to five detached leaf replicates per growth stage. Figure $2 \mathrm{C}$ shows colony number ( $y$-axis) differences between first and third leaves. Figure 2D similarly shows colony size (y-axis) differences between first and third leaves. Error bars are standard errors for five replicates (C) and (D). 

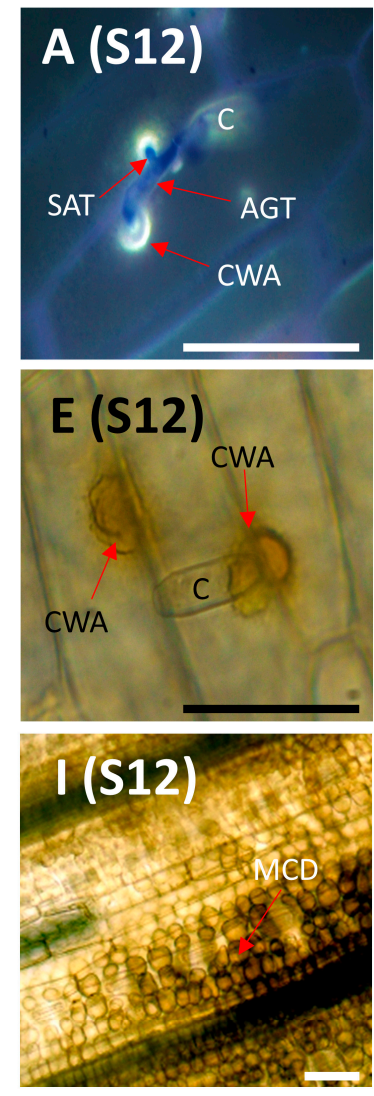
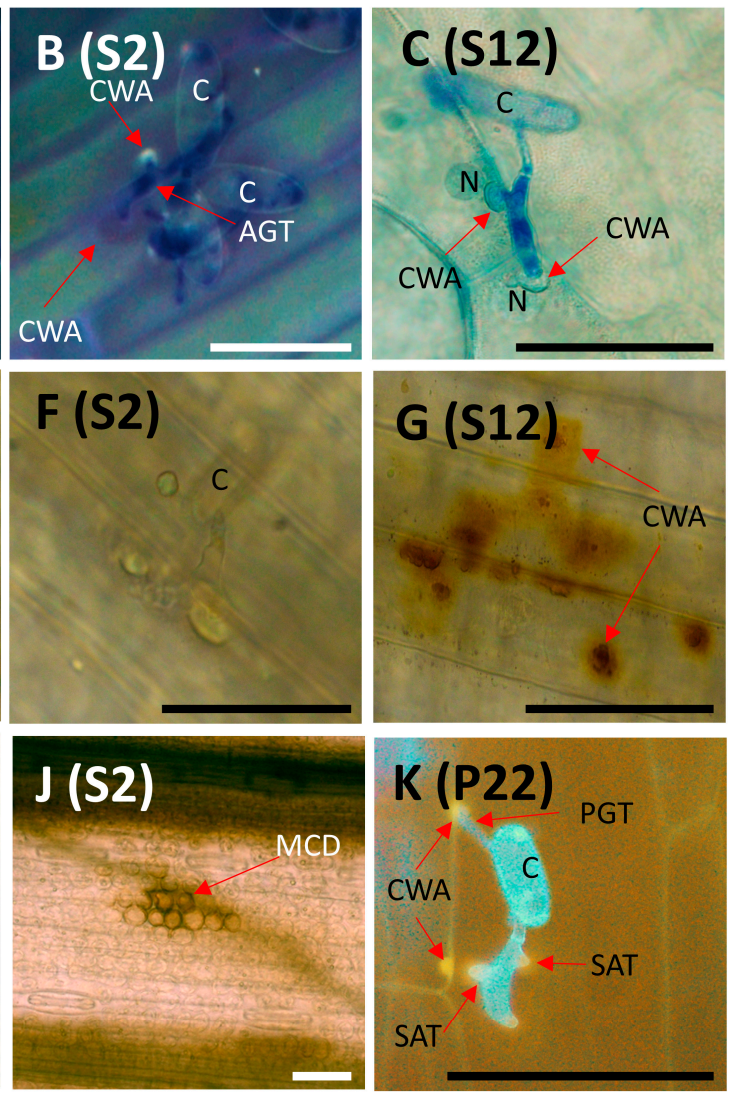
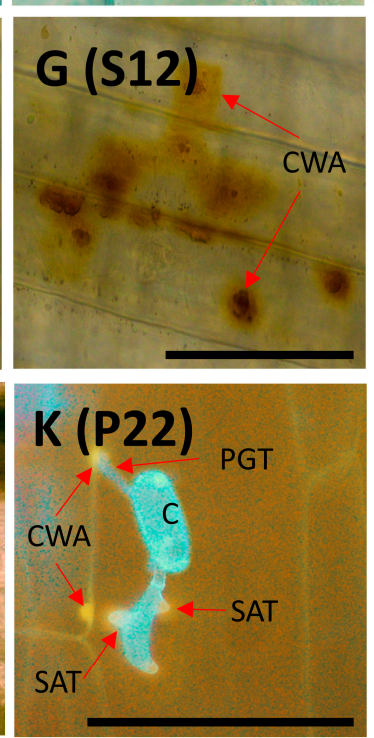
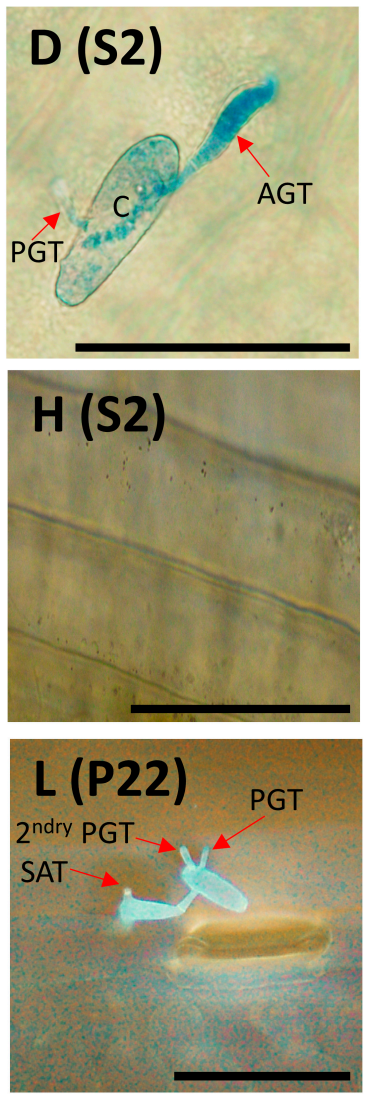

Figure 3. Microscopic images of Baudin near-isogenic line (NIL) responses to attempted penetration by Blumeria graminis f sp. hordei. (A,C,E, G,I) are S12 (mlo-11). (B,D,F,H,J) are S2 (mlo-11(cnv2)). (K) and (L) are NIL P22 (mlo-5). (G-J) were not inoculated. (A,B) were double-stained with aniline blue and Evans blue for visualizing cell-wall appositions (CWAs). (C,D) were stained with trypan blue. (E-J) were stained with 3,3'-diaminobenzidine (DAB). (K,L) were triple-stained with aniline blue, calcofluor white, and Evans blue and show Bgh phenotypic responses in P22. Abbreviations: AGT, appressorial germ tube; C, conidia; CWA, cell-wall apposition; MCD, mesophyll cell death; N, nucleus; PGT, primary germ tube; SAT, secondary appressorial tube. Scale bar represents $50 \mu \mathrm{m}$.

\subsection{Small RNA (sRNA) RNA-Directed Mlo Methylation is Associated with Histone Modification}

sRNA sequence alignments to $M l o$ showed that wild-type Mlo had very few sRNA reads (an average of 60, Figure 4) which were sense-stranded. For S2, 274 reads of sRNA mapped to the mlo-11 tandem repeats, as well as Mlo. Of these, the largest class was $24 \mathrm{nt}$ (61 reads) with the majority sense-stranded. There is a peak of alignments in a region of antisense small RNA at 300 to $600 \mathrm{bp} 5^{\prime}$ to the Mlo ATG with a TATA motif, indicative of a TATA box. The sequence within this peak lies at the start of the main block of $5^{\prime}$ untranslated region (UTR) methylation in a CHH context, in addition to an isolated $3^{\prime}$ region shared with S12. Standard mlo-11 showed significant differences to mlo-11(cnv2); 1188 reads (880 of $24 \mathrm{nt}$ ) sRNA mapped to this variant and, in contrast to Mlo and mlo-11(cnv2), most of the $24 \mathrm{nt}$ sRNA were antisense. The alignment peak region around the TATA was larger than in mlo-11(cnv2), from around 150 to $600 \mathrm{bp} \mathrm{5'}$ to ATG, coincident with greater methylation. Standard mlo- 11 also showed substantial alignment antisense peaks that correspond to the start and end of the mlo-11 subunit repeat. 


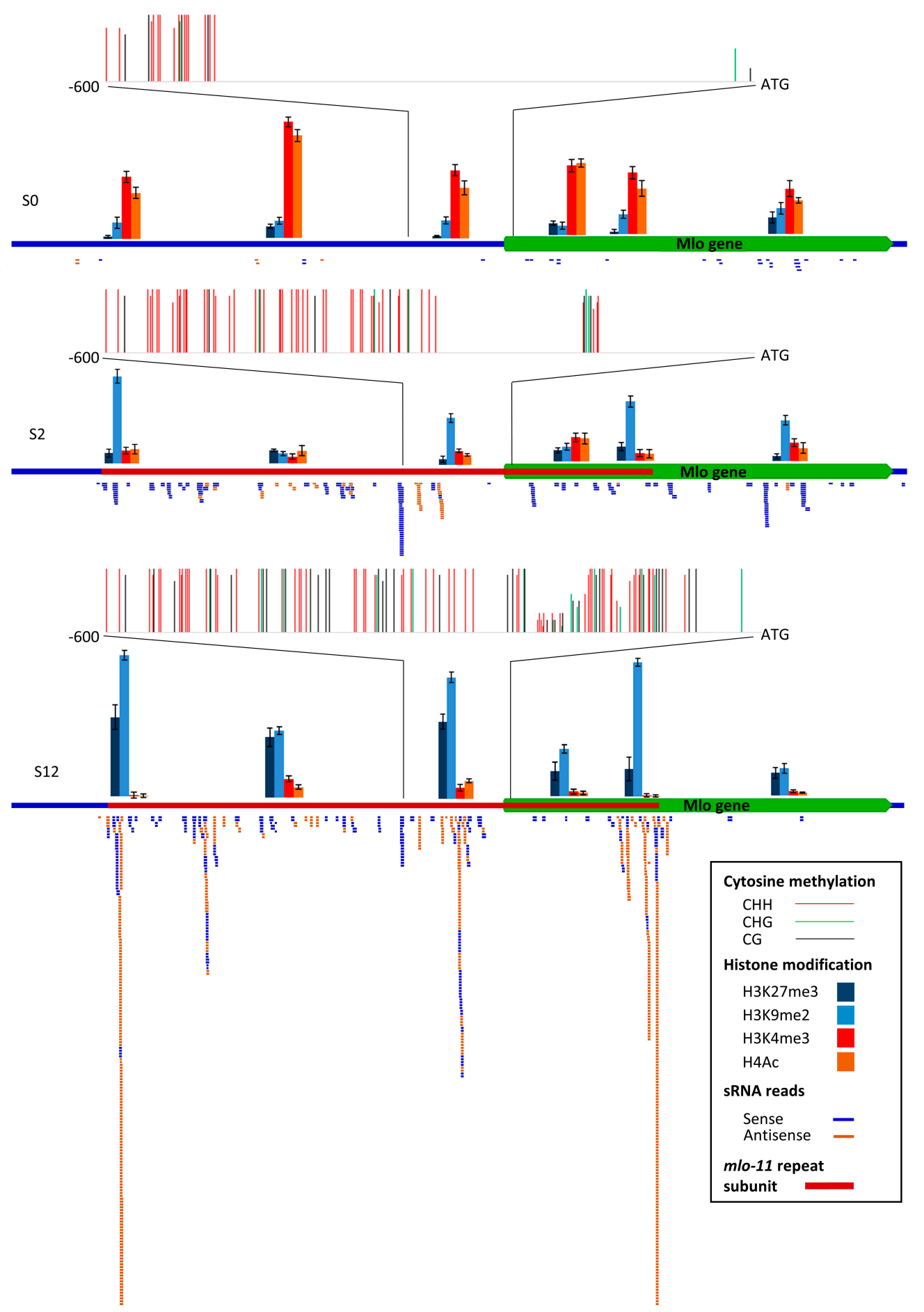

Figure 4. Evidence for small RNA (sRNA)-directed histone modifications at the mlo-11 subunit between Mlo wild type control (S0) and Baudin NILs containing mlo-11(cnv2) (S2) and standard mlo-11 (S12). The top panel shows methylation status of a $600 \mathrm{bp}$ region determined by bisulphite sequencing. Red depicts methylation in the $\mathrm{CHH}$ context, green the CHG context, and black the CG context (where $\mathrm{H}=\mathrm{A}, \mathrm{T}$, or $\mathrm{C}$ ), scaled relative to presence in 10 sequenced replicates. The majority of cytosine methylation is in the $\mathrm{CHH}$ context. The middle panel shows levels of histone modifications in six regions determined by chromatin immunoprecipitation (ChIP) PCR. Each histone modification class is scaled is relative to the anti-H3 histone background control. The bottom panel shows sRNA reads mapped across the mlo- 11 repeat subunit. 
Overall, the methylation levels, sRNA antisense read alignments, and histone modifications between the three NILs (Figure 4) support a correlation with Mlo expression differences (Figure 5), with the number of sRNA mapped regions higher in S12 than S2, including across the promoter region, and an absence from Mlo exons 6-12, which are not present in the mlo-11 repeat units (Figure 4).

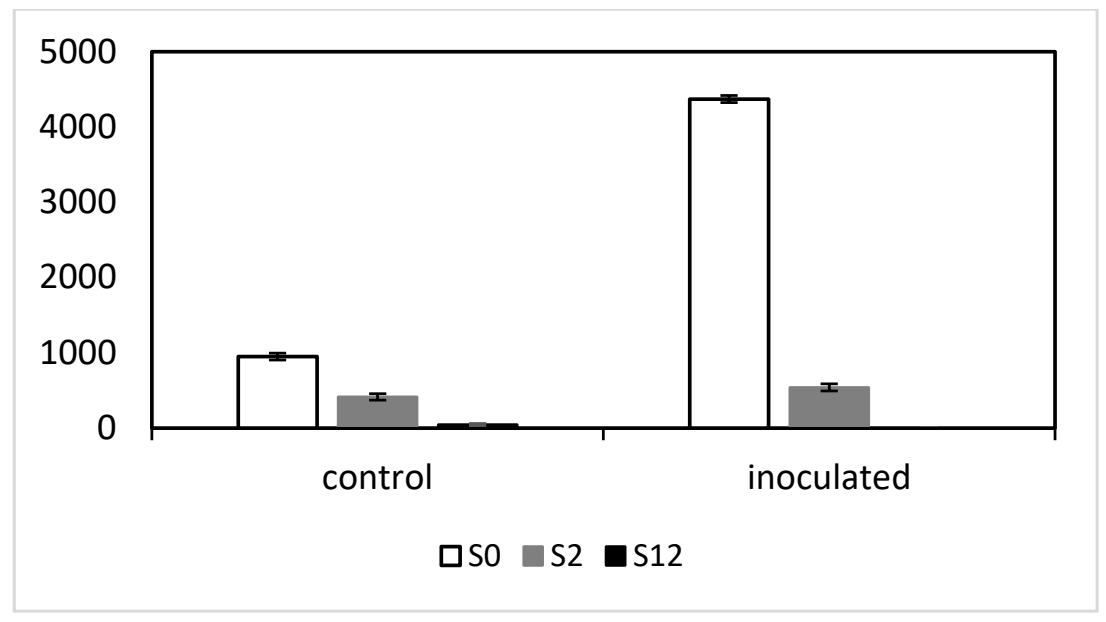

Figure 5. Number of messenger RNA (mRNA) reads mapped to Mlo exons 6 to 12 ( $Y$-axis) for Baudin NILs containing mlo-11(cnv2) (S2), mlo-11 (S12), and wild-type Mlo (S0). Suppressed expression for exons 6 to 12 (not present in the mlo-11 tandem repeats) in S12 is shown, with intermediate suppression in S2, consistent with the epigenetic changes shown in Figure 4. Read counts are averaged across three replicates. The $Y$-axis shows the number of mRNA reads mapped.

Bisulphite sequencing of a $600 \mathrm{bp}$ region upstream of the Mlo start codon showed that, $5^{\prime}$ to the first 200 bp region next to the Mlo ATG, most methylation is in the CHH context (Figure 4). In S12, the first $50 \mathrm{bp}$ and from 240 to $270 \mathrm{bp} 5^{\prime}$ to ATG is nonmethylated, and 130-200 bp 5' to the Mlo start codon is partially methylated (around $40 \%$ ). The remaining sequence is highly cytosine methylated $(>90 \%)$. For S2 at around 300 to $6005^{\prime}$ from the start codon, including the position of a peak of aligned antisense sRNA reads, most cytosines are highly methylated.

Since DNA methylation can be linked to both histone modifications and regulation of gene expression [12], we examined modification in six Mlo regions. Five of these regions were in the mlo-11 repeat subunit. These were the repeat start region, $5^{\prime}$ to the promoter region, the promoter region in the vicinity of the TATA box and the $5^{\prime}$ UTR region of the truncated Mlo gene, and exon 5 before the repeat truncation site (Figure 4). For a region representing the wild-type Mlo gene. we used the Mlo exon 9. Chromatin immunoprecipitation (ChIP) followed by qPCR indicated that the key epigenetic markers histone $\mathrm{H} 3$ lysine 9 dimethylation (H3K9me2) and H3 Lysine 27 trimethylation (H3K27me3), usually linked to DNA repeat inactivation [24], were high in the promoter region in S12, very low in S0, and intermediate in S2, complementing the bisulphite sequencing. The regions at the start and end of the mlo-11 repeat subunit showed highest H3K9me2 and H3K27me3 levels in S12, matching antisense sRNA sites, together with a central region (R3). In S2, a similar pattern was evident but with the central region $\mathrm{R} 3$ showing highest repression marks. Histone $\mathrm{H} 4$ acetylation and $\mathrm{H} 3 \mathrm{~K} 4$ trimethylation, related to gene activation, showed an inverse correlation with $\mathrm{H} 3 \mathrm{~K} 9 \mathrm{me} 2$ and H3K27me3, with very low levels in S12 and intermediate levels in S2.

\subsection{Exon-Specific Expression Differences between the mlo NILs}

The colony count and IT results positively correlated with wild-type Mlo RNA levels among the five NILs. All RNA reads mapped to Mlo are shown in Figure S2 (Supplementary Materials). However, most of the reads for S2 and S12 only mapped to Mlo exons 1-5 contained in the mlo-11 tandem repeat, as well as the $3000 \mathrm{bp}$ promoter region before ATG, with the reads truly determined as wild-type Mlo 
only mapping to Mlo exons 6-12 (Figure 5). For P22, since the start codon ATG is point mutated [17], transcription occurs and no functional MLO protein is formed. In S12, very low levels of Mlo were evident following inoculation and in noninoculated controls. In the absence of pathogen, wild-type Mlo reads in S2 were about half the levels seen in S0. Following infection, Mlo in S2 did not increase significantly and was only one-seventh of the level of S0 which increased dramatically, a characteristic reported previously [3].

\subsection{Whole-Genome Expression Comparisons of the Mlo NILs}

A principal component analysis (PCA) of whole-genome expression during Bgh infection further resolved phenotypic differences between all the NILS in this study (Figure 6). On PC1 a variance of $70 \%$ was explained by the different genetic backgrounds of the two cultivars Baudin and Pallas. On PC2, a variance of $21 \%$ represented different alleles and treatments. Uninfected controls for P18, S0, and S2 all grouped together, P22 and S12 uninfected controls clustered with their respective wild-type Mlo-infected samples, and infected S2, S12, and P22 samples grouped at the bottom end of PC2. Uninfected S12 and P22 appear to have similar expression patterns to infected but compatible responses in $\mathrm{S} 0$ and $\mathrm{P} 18$, respectively.

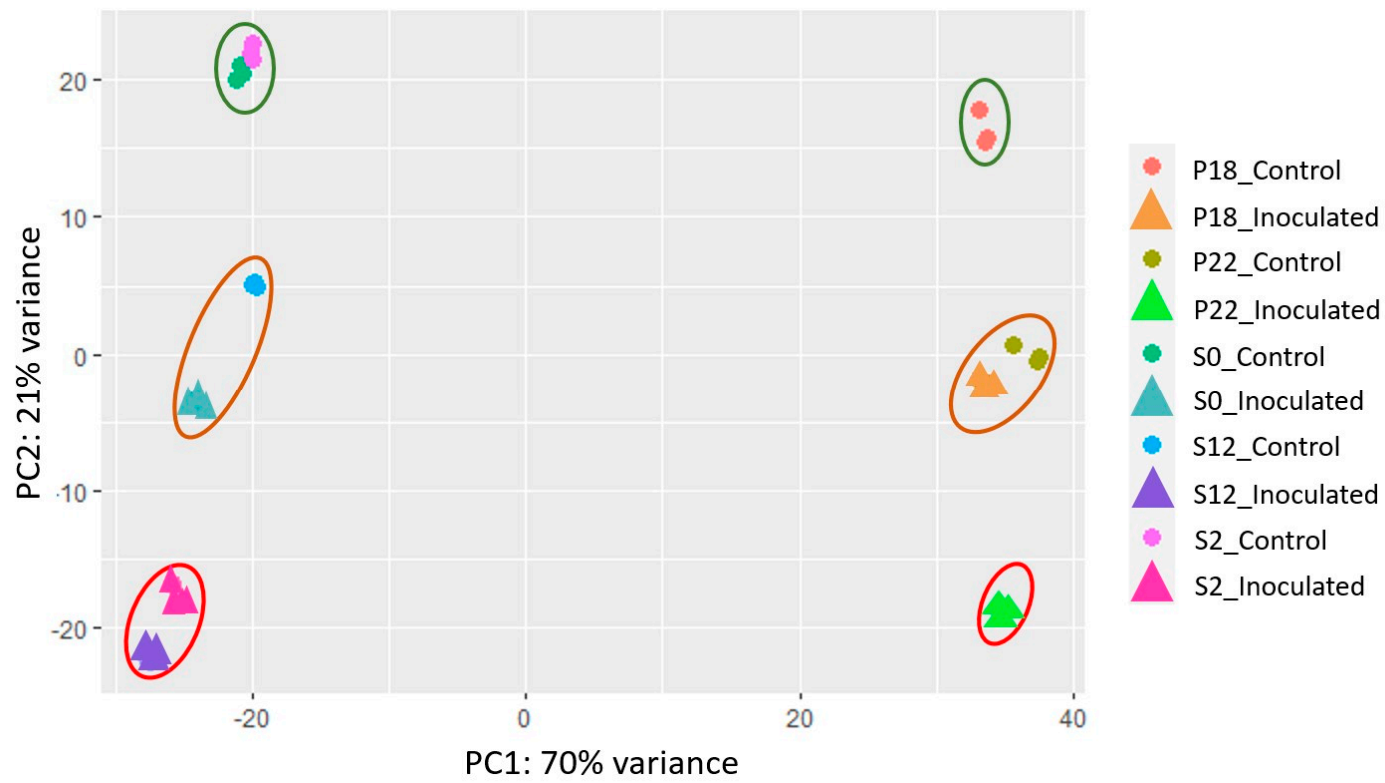

Figure 6. Principal component analysis (PCA) of whole-genome expression for Pallas NILs containing mlo-5 (P22) and Mlo (P18) and Baudin NILs containing mlo-11 (S12), mlo-11(cnv2) (S2), and Mlo (S0) in control and Bgh inoculated samples. PC1 represents the main variance (72\%) between Baudin and Pallas genotypes, while PC2 shows the variation (20\%) between Mlo or mlo alleles and treatments (control versus Bgh inoculated).

Whole-genome expression pairwise correlation coefficients for the Baudin and Pallas NIL samples were analysed (Figure 7 and Figure S3, Supplementary Materials, respectively). The correlation for whole-genome expression between the Baudin NIL replicates showed that S0 and S2 noninfected controls were significantly correlated, but negatively correlated to the S12 control and S0, S2, and S12 infected samples. S2 and S12 infected samples were highly correlated (Figure 7). Whole-genome expression pairwise correlation coefficients and significance for the Pallas NILs showed significant correlations between P18 inoculated and control and P22 control samples, but negative correlations with P22 inoculated samples (Figure S3, Supplementary Materials). 

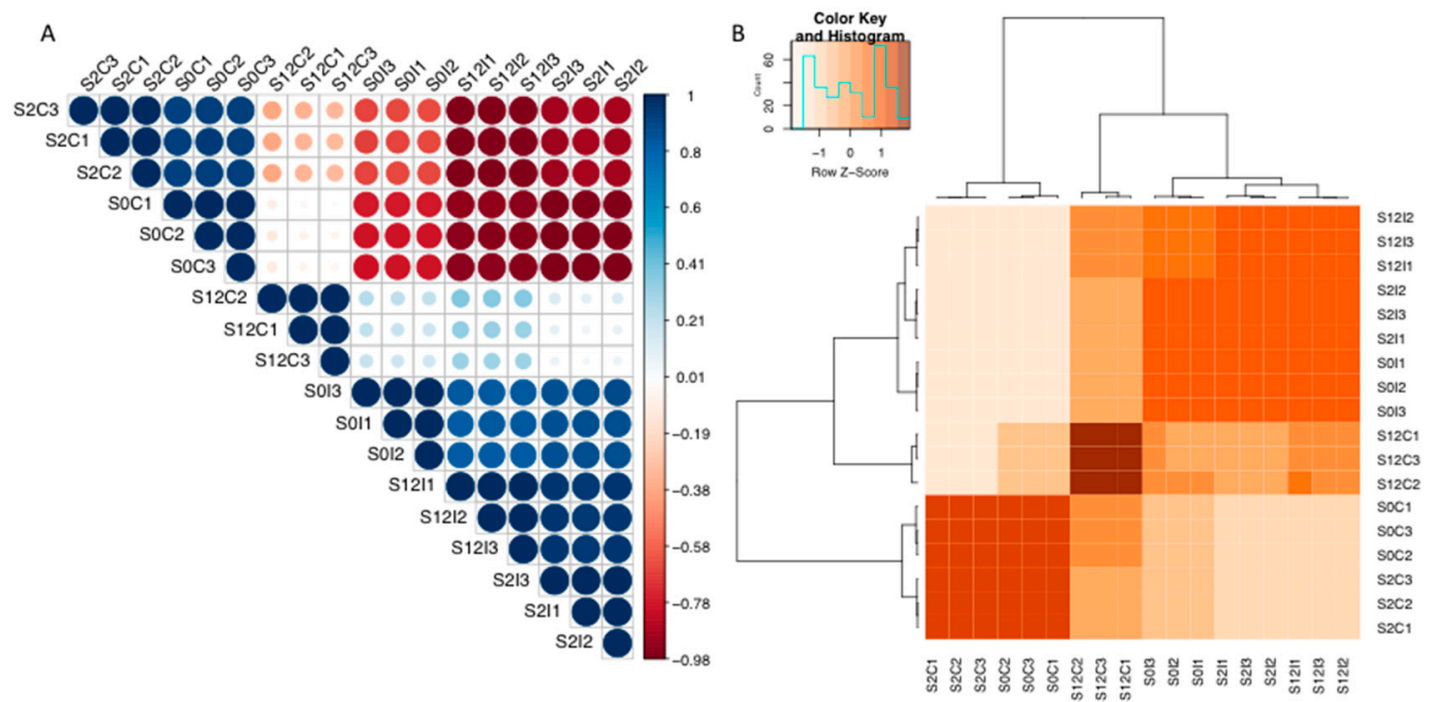

Figure 7. Baudin NILs mlo-11(cnv2) (S2), mlo-11 (S12), and Mlo wild-type control (S0) sample pairwise correlation coefficient plots of gene expression in Bgh inoculated (I) and noninoculated controls (C) for replicates 1-3. (A) Positive correlation coefficient (blue) and negative correlation (red) with dots sized by relative correlation $p$-values. (B) Heatmap samples are clustered by correlation coefficients. A count histogram is shown in the colour key for values.

\subsection{Differential Gene Expression Analysis and Gene Ontology Enrichment between NILs}

Among the Baudin NILs, a total of 3470 genes were identified as significantly regulated (Table S1, Supplementary Materials). The Pallas lines exhibited a total of 3312 significantly regulated genes (Table S2, Supplementary Materials). Expression profiles of the Baudin NILs samples (S0, S2, and S12) for the topmost 100 regulated genes showed that a majority of genes were induced in S2 and S12 during infection (Figure S4, Supplementary Materials) compared to the remaining samples. Furthermore, the Baudin NILs clustered into two major groups represented by Bgh inoculated and control samples, with a closer grouping for S0 and S2 when noninfected and a closer grouping of S2 and S12 when inoculated by Bgh (Figure S4, Supplementary Materials). In the Pallas NILs samples (P22 and P18), the majority of genes were induced in P22 during infection as compared to the remaining samples. Furthermore, the P18 infected grouped with P22 noninfected and noninfected P18 showed the lowest overall expression levels (Figure S5, Supplementary Materials).

Among the Baudin NILs, expression data for Bgh infected samples and noninfected samples (Figure 8) illustrated that S2 oscillates between a wild-type Mlo-like resting state when uninfected and S12 levels of expression when infected, and that S12 maintains elevated states of gene expression under both conditions. Six genes notably downregulated for S2 when infected (Figure 8) were represented by three oxidoreductases, one of which (HORVU1Hr1G057860) was downregulated in all three NILs and is annotated as a cytokinin oxidase/dehydrogenase. The remaining two oxidoreductases were downregulated in all three NILs, but to a greater extent in S12 and S2, and are annotated as a monooxygenase (HORVU4Hr1G066230) and a cytokinin oxidase/dehydrogenase (HORVU7Hr1G118130). Two genes specifically downregulated in S2 relative to S12 included a protein involved in catabolism (HORVU3Hr1G006440) and a laccase (HORVU1Hr1G008360). The remaining gene of unknown function (HORVU4Hr1G079440) was downregulated in S2 and to a lesser extent S12, but upregulated in S0. 
A Inoculated

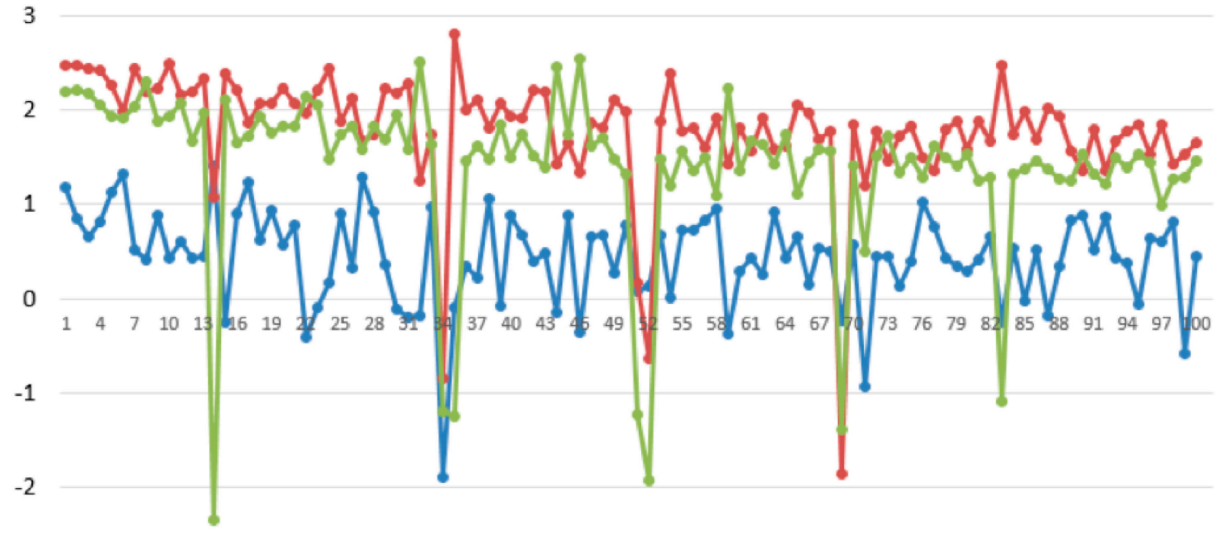

$-3$

$\rightarrow \mathrm{SO} \rightarrow \mathrm{S} 12 \rightarrow \mathrm{S} 2$

B

Control

4

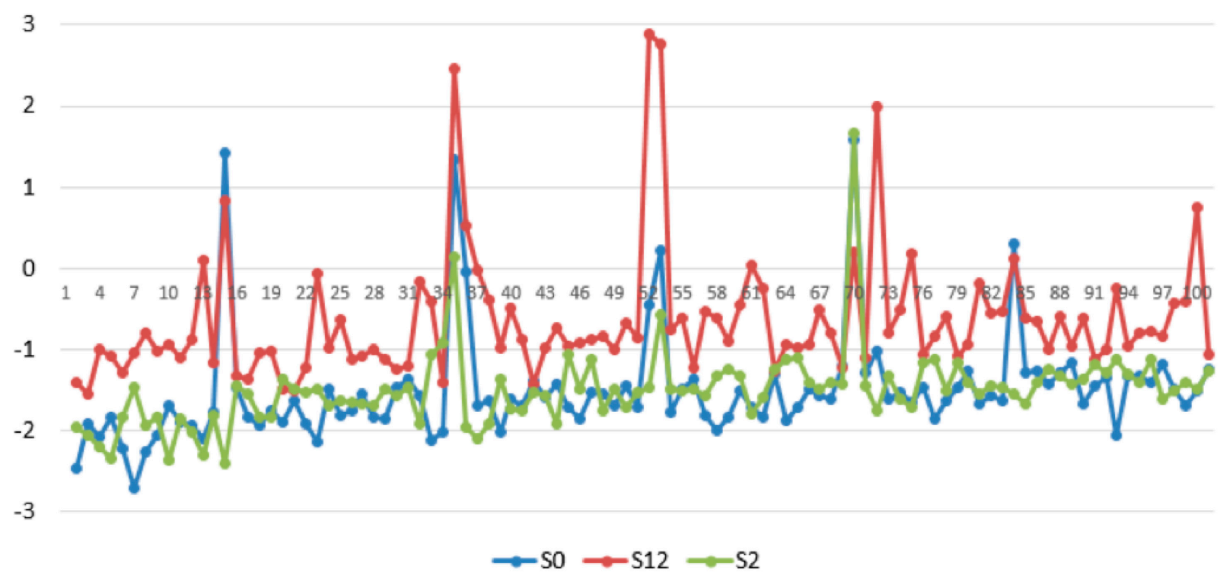

Figure 8. Top 100 differentially expressed genes (X-axis) for Baudin NILs containing S12 (mlo-11), S2 (mlo-11(cnv2)), and S0 (Mlo) during (A) infection by Bgh and (B) noninoculated control samples, represented through variance-stabilizing transformation (VST) $z$-score ( $Y$-axis).

The top 100 regulated Baudin NIL genes contained 24 biological process Gene Ontology (GO) terms containing 63 genes significantly enriched at a $p$-value $\leq 0.01$ (Figure S6, Supplementary Materials). These included response to parasitic fungus $\left(p\right.$-value $\left.=3.35 \times 10^{3}\right)$, regulation of salicylic acid biosynthesis $\left(p\right.$-value $\left.=3.35 \times 10^{3}\right)$, and negative regulation of gibberellic acid $\left(p\right.$-value $\left.=3.30 \times 10^{-5}\right)$. A total of 16 molecular function GO terms were significantly enriched by 62 genes, which included catalytic activity $\left(p\right.$-value $\left.=8.20 \times 10^{-5}\right)$, hydroperoxide dehydratase $\left(p\right.$-value $\left.=9.50 \times 10^{-6}\right)$, and calcium ion binding $\left(p\right.$-value $\left.=1.20 \times 10^{-4}\right)$ activities.

The top 100 regulated Pallas genes (Figure S7, Supplementary Materials) contained 54 biological process GO terms containing with 61 genes significantly enriched ( $p$-value $\leq 0.01$ ). These included chitin catabolism ( $p$-value $\left.4.28 \times 10^{-5}\right)$, protein phosphorylation ( $p$-value $\left.3.18 \times 10^{-7}\right)$, and defence response ( $p$-value $2.22 \times 10^{-5}$ ). A total of 39 molecular function GO terms were significantly enriched by 66 genes, which included carbohydrate binding $\left(p\right.$-value $\left.1.78 \times 10^{-7}\right)$, and protein kinase $(p$-value $2.04 \times 10^{-8}$ ) and chitinase ( $p$-value $2.72 \times 10^{-5}$ ) activities. Additional differences between mlo-11 and mlo-5 NIL GO term biological processes appeared to be those involving innate immune responses and aromatic amino-acid metabolism in mlo-5. 


\subsection{A WRKY Transcription Factor Binding Site Is Present in Highly Expressed Genes}

The promoter region $1000 \mathrm{bp} 5^{\prime}$ from the start codons of the top 100 differentially expressed (DE) genes were extracted and analysed via the MEME software suite [25]. This identified a transcription factor binding motif shared between 16 genes differentially expressed and related to pathogenesis and defence responses. The motif was most similar to the WRKY TF W-box motif (T)TGAC(C/T) [26,27]. Instead of the second thiamine, a guanine is present to give a core sequence GGACT, with an extended consensus of CCGGGACTAA (Figure S8A, Supplementary Materials). Notably, of the top 20 most DE genes, 10 contained this motif within $1000 \mathrm{bp}$ of the start codon (Figure S8B, Supplementary Materials). Gene annotations and their GO terms indicated that these genes are all involved in defence responses. Two of these genes (WRKY33_HORVU1Hr1G070250 and WRKYGK_HORVU1Hr1G080300) were categorised as encoding WRKY TF proteins. A total of 102 barley genes encoding WRKY motifs present in IBSC assembly v2 [28] were investigated and, of those, only the two genes identified here possessed the CCGGGACTAA motif.

\subsection{Quantitative PCR of Highly Regulated Genes Associated with the New TF Motif}

The RNA-seq read count for each of the individual 10 genes associated with an upstream W-box motif were examined and verified by qPCR (Figure S9, Supplementary Materials). The two WRKY-like genes, WRKY33_HORVU1Hr1G070250 and WRKYGQ_HORVU1Hr1G080300, exhibited different expression patterns between inoculated and control samples. Both were most highly expressed in P22 (mlo-5) in control samples, particularly WRKYGQ_HORVU1Hr1G080300. However, on infection, all samples showed similar levels of WRKYGQ_HORVU1Hr1G080300 induction, suggesting that the gene is not specifically associated with mlo powdery mildew resistance. In contrast, WRKY33_HORVU1Hr1G070250 was most highly expressed in S12 and S2 samples on infection, followed by P22.

The peroxidase HORVU5Hr1G097270 expression increased on infection in proportion with the strength of the mlo mutation (P22 followed by S12 and S2). In control samples, peroxidase HORVU5Hr1G097270 was most highly expressed in P22. The oxidase HORVU7Hr1G118130 was repressed in all samples on infection, and most noticeably in S12 and P22, while, in the wild-type $M l o$ lines, it incongruously increased in S0 but not P18. This difference may represent $B g h$ promoting growth in a susceptible line or a genotype-specific response in SO.

HORVU0Hr1G005300 is orthologous to the Downy mildew resistance 6 gene (DMR6, At5g24530) involved in the defence response to oomycetes and is a dioxygenase domain-containing protein. The gene was most highly expressed in infected and control samples of S12. Expression was not associated with the mlo mutation or wild-type Mlo in the remaining NILs, suggesting that this gene is not involved in mlo powdery mildew resistance.

Of the remaining genes, three were pathogenesis-related (PR) such as homologues PR1_HORVU5Hr1G056040, PR1a_HORVU5Hr1G05595 and PRB1-2_HORVU7Hr1G033530, a thaumatin (HORVU5Hr1G051970), and a chitinase (HORVU1Hr1G052430). All were most highly expressed on infection in P22, followed by S12 then S2.

\section{Discussion}

The mlo-11(cnv2) allele represents an example of modification of Mlo gene expression which refines downstream gene expression in a manner that both provides basal defence gene expression levels similar to wild-type Mlo in noninfected plants and allows overall expression levels similar to standard mlo-11 when infected. Similar cytological differences were observed between S2 and S12 variants in the cv Baudin NILs compared to the donor lines described in Ge et al. [9], namely, a lack of spontaneous CWA and mesophyll cell death in S2, together with less prominent CWAs and fewer secondary appressorial and primary growth tubes in seedling leaves. Young S2 NIL plants also showed slightly delayed onset of powdery mildew resistance compared to S12, confirming apparent developmental onset found previously $[9,29]$. The more obvious CWAs in S12 may be expected 
to contribute to the earlier complete resistance, although, for effective penetration resistance, CWA polysaccharide composition is considered essential [30].

The sRNA data indicated that antisense $24 \mathrm{nt}$ reads exclusively led to suppressed Mlo expression in the mlo-11 variants. This is consistent with a study by Hackenberg et al. [31], who found that this class of sRNA in barley, in common with other plant species, is mostly associated with repeats. The 24 nt RNA represents heterochromatic small interfering RNAs (siRNAs) [32] which form from double-stranded RNAs derived from a range of sources that produce RNA sense-antisense pairs, including the mlo-11 subunit tandem repeats in this study. siRNA read alignment data suggested peaks of antisense RNA in the Mlo promoter and, therefore, we extended previous bisulphite sequencing of the region [9] to a larger $600 \mathrm{bp}$ section. This showed that antisense RNA alignment sites were highly methylated, indicative of their key role in producing siRNA, and that $\mathrm{CHH}$ methylation predominated. In plants, siRNAs are known to be associated with repressive chromatin modifications via 5-methyl cytosine, especially at asymmetric $\mathrm{CHH}$ sites [12].

Histone immunoprecipitation followed by qPCR indicated that the repression marks H3K9me2 and H3K27me3 methylation levels were intermediate in S2 compared to S12 and S0, complementing the bisulphite sequencing. Interestingly, in $\mathrm{S} 12 \mathrm{H} 3 \mathrm{~K} 9 \mathrm{me} 2$ and H3K27me3, methylation levels were highest in regions at the start and end of the mlo-11 repeat subunit, together with a central region. S12 appeared devoid of the activation marks H4Ac and H3K4me3, with S2 exhibiting moderate levels although lower than H3K9me2 and H3K27me3. This observation explains the almost complete Mlo repression in S12 compared to modest expression in S2 and confirms the inference of Buschges et al. [17] that the Mlo phenotype is dependent on the extent of MLO protein inhibition.

Examination of expression profiles for the most highly regulated genes between the Baudin NILs revealed similar gene expression patterns for infected S2 and S12 samples and between S0 and S2 controls. S2 powdery mildew-related gene expression is, therefore, moderated until stimulated by Bgh infection. This was also the case for global gene expression by PCA, where the variability between these samples was markedly small, indicating the widespread but conserved effect of Mlo inhibition.

Two highly expressed WRKY TFs appear to contain a modified promoter W-box motif, with the consensus sequence CCGGGACTAA rather than (T)TGAC(C/T) [26], although this remains to be experimentally verified. WRKY gene promoters containing W-boxes may act as activators and repressors and interact with a range of proteins, including other TFs, and are extensively involved in auto- and cross-regulation [27]. One of the TFs, WRKYGQ_HORVU1Hr1G080300, was induced to a similar level in all samples. However, WRKY33_HORVU1Hr1G070250 was associated with mlo resistance, particularly mlo-11 resistance. The contribution of this TF to powdery resistance and whether null or alternative alleles naturally occur may have significance in future crop breeding for host resistance.

Pathogen challenge in plants generally causes downregulation of auxins and gibberellins involved in growth in a trade-off with defence responses [19]. The upregulation of salicylic acid biosynthesis and downregulation of gibberellic production were particularly evident in the top 100 most regulated genes for the mlo-11 NILs compared to mlo-5. Other main differences in NIL GO term biological processes between $m l o-11$ and $m l o-5$ NILs appeared to be those involving innate immune responses and aromatic amino-acid metabolism in $m l o-5$, with the increased aromatic amino-acid metabolism in $m l o-5$ plants presumably leading to higher levels of phenolic compounds.

The mlo NILs showed greater changes in relative abundance of nicotinamide adenine dinucleotide phosphate (NADPH) oxidases (NOXs) and peroxidases (POXs) during infection. Powdery mildew defence involves reinforcing of the cell wall, while growth, by contrast, often necessitates the loosening of the cell wall to allow cellular expansion. Neuser et al. [20] showed that the TF HBl1 differentially regulates the expression of these two responses through ROS homeostasis within the apoplast (the space between plant cell walls). In growing plants, HBI1 configures apoplastic ROS levels that support growth by activating specific NOX genes and repressing specific POX genes. When challenged by a pathogen, HBI1 is deactivated, reversing the activation of NOX genes and repression of POX genes, 
resulting in increased apoplastic ROS levels, repressing growth but increasing disease resistance. This is evident in peroxidase_HORVU5Hr1G097270 and oxidase_HORVU7Hr1G118130, where the POX gene is upregulated within the mlo resistant NILs upon infection whereas NOX is repressed (Figure S9, Supplementary Materials). ROS are directly antimicrobial and lead to protein cross-linking and CWA formation, while they also have roles in cellular signalling associated with the induction of defence gene expression and the hypersensitive response (reviewed in [33]). The effects of these changes are most extreme in mlo-5, followed by S12 then S2. The toxic effects of the ROS and phenolic compound levels in mlo-5 plants plausibly contribute to their generally senescent phenotype. ROS levels in S12 plants appear to avoid such an extreme effect but still induce spontaneous cell death, while S2 plants show the lowest perturbation.

This study resolves the underlying basis for the mlo-11(cnv2) phenotype, which is governed by intermediate defence gene expression levels that correlate with the level of antisense $24 \mathrm{nt}$ siRNA mediated Mlo suppression. The original landrace containing mlo-11(cnv2), HOR2543, has no collection information but is thought to have been collected early in the last century alongside much of the ex situ Ethiopian germplasm. The variant allele potentially represents a relatively recent mutational event since the majority of Ethiopian mlo mutants are standard mlo-11. As a test of natural fitness, the variant's persistence or selection in new landrace collections may define the evolutionary outcomes of the allele.

\section{Materials and Methods}

\subsection{Plant Materials}

Near-isogenic lines were created at the speed breeding facility at the University of Queensland. Cultivars Westminster (mlo-11) and Eth295 (HOR2547, mlo-11(cnv2)) were backcrossed to a powdery mildew susceptible cultivar containing wild-type Mlo, cv Baudin (S0), for five generations (average NIL cv Baudin content equivalent to $96.87 \%$ ) to give NILs S2 and S12, respectively. PCR primers to identify mlo-11 progeny during introgression and cleaved amplified polymorphic sequences (CAPS) primers to distinguish Baudin Mlo and mlo-11(cnv2) alleles are presented in Table S3 (Supplementary Materials). Pallas NILs [21] P18 (powdery mildew susceptible, wild-type Mlo) and P22 (mlo-5) were obtained from Ryan Fowler (Department of Agriculture and Fisheries, Queensland, Australian).

\subsection{Bgh Culture and Plant Growth Conditions}

A monoconidial West Australian Bgh isolate, Chi-001, was propagated on detached leaf sections of $\mathrm{cv}$ Baudin inserted into $50 \mathrm{mg} \cdot \mathrm{L}^{-1}$ benzimidazole agar plates and grown in a Contherm Biosym $6200 \mathrm{CP} 4$ incubator (Contherm Scientific Ltd., Petone, New Zealand) at $16{ }^{\circ} \mathrm{C}$ and $10{ }^{\circ} \mathrm{C}$ under a $12 \mathrm{~h}$ light and dark cycle, respectively. Barley plants for cytology, seedling detached leaf infection assays, RNA-seq, and ChIP assays were grown in vermiculite fertilised with Thrive all-purpose soluble plant food (Yates, Clayton, Victoria, Australia) and under light shelves at $300 \mu \mathrm{mol} \cdot \mathrm{m}^{-2} \cdot \mathrm{s}^{-1}$. Plants for whole-plant infection assays were grown in soil with Nitrophoska Special S (EuroChem Antwerpen $\mathrm{NV}$, Antwerpen, Belgium) in a controlled temperature room $\left(18-22^{\circ} \mathrm{C}\right)$ with a $12 \mathrm{~h}$ photoperiod at $450 \mu \mathrm{mol} \cdot \mathrm{m}^{-2} \cdot \mathrm{s}^{-1}$. A minimum of three replicate plants were used for each accession and treatment, unless specified in the results. Detached leaves and whole plants were inoculated in a settling tower [34] and scored on the 0-4 infection type (IT) scale of Mains et al. [35], where $0=$ no visible symptoms, 1 = sparse mycelial development with no sporulation, $2=$ mycelial present with very few spore chains, $3=$ moderate mycelial development and discrete lesions with sporulation, and $4=$ amorphous mycelial development and abundant sporulation.

\subsection{Cytology and Detached Leaf Inoculations}

$B g h$ infected leaves were sampled at $72 \mathrm{~h}$ post infection (hpi) by taking $0.5 \mathrm{~cm}^{2}$ leaf segments from 10 independent leaves per genotype. Triple staining with Evans blue, aniline blue, and calcofluor white, 
double staining with staining with Evans blue and aniline blue, and DAB ( $3,3^{\prime}$-diaminobenzidine) and trypan blue were described previously [9]. Fluorescence for double- and triple-stained samples was detected using the Zeiss filter set 38 (Carl Zeiss Microscopy, White Plains, NY, USA: BP 470/40 nm; FT $495 \mathrm{~nm}$; BP 525/50 nm).

Powdery mildew colony counts and size measurements of individual genotypes were performed with detached leaves using five replicates per growth stage at the first- and third-leaf stages. Leaves were severed, inserted onto benzimidazole agar plates, and inoculated using a powdery mildew settling tower [34]. Bgh colonies were counted within $1 \mathrm{~cm}^{2}$ leaf sections at 14 days post inoculation. Colony diameters were estimated for 10 colonies per replicate detached leaf.

\subsection{Whole-Genome RNA and Small RNA Sequencing}

For each barley line (S0, S2, S12, P18, and P22), leaf tissue was sampled from three biological replicates at $48 \mathrm{hpi}$ for infected samples together with noninoculated controls. All plants were grown in a high-humidity chamber under the same conditions. Leaf tissue was sampled from each accession at the third-leaf stage. Total RNA was extracted using an RNeasy Plant Mini Kit (Qiagen, Hilden, Germany) following the manufacturer's protocol. For RNA-seq, RNA libraries were made with an Illumina TruSeq RNA v2 kit and sequenced with 150 bp paired-end reads on an Illumina HiSeq 2500 platform (Illumina Inc., San Diego, CA, USA) at the Novogene Institute (Beijing, China) using stranded protocols. The Illumina CASAVA1.8 pipeline was used to generate sequence data. Small RNA (sRNA) sequencing was performed by the Novogene Institute on an Illumina HiSeq 2500, with read cleaning of sequencing adapters and non-sRNA contaminants. The RNA-seq read data were deposited at DDBJ/ENA/GenBank under accession PRJEB39864 and sRNA-seq data were deposited at DDBJ/ENA/GenBank under accession PRJEB40118.

\subsection{RNA-seq Analysis}

RNA-seq reads were quality checked with FastQC v0.11.5 [36] and trimmed with a Phred score $\geq 30$ using Trimmomatic v0.33 [37] with HEADCROP:12, ILLUMINACLIP:TruSeq-PE.fa:2:30:5, LEADING:10, TRAILING:10, SLIDINGWINDOW:4:25, and MINLEN:50 parameters. Quality trimmed reads were aligned to the International Barley Sequencing Consortium (IBSC) barley genome version 2.43 [28] using the RNA-seq aligner Star version 2.7.0e [38] with quantMode equal to GeneCounts. Gene expression counts were calculated and guided by the barley genome version 2.43 gene annotations. Normalisation, gene dispersion estimates, and differential gene expression were performed using $\mathrm{R}$ v3.5.1 package DESeq2 version 1.22.2 [39]. A sample principal component analysis (PCA) was conducted on expression data using variance-stabilizing transformation (VST) across the five lines (Baudin (S0), S2, S12, P18, and P22) for inoculated and noninoculated samples. The most significant differential gene expression between the samples was set at $\geq 2$-fold change and a Benjamini-Hochberg (BH) adjusted $p$-value (false discovery rate) $\leq 0.05$ [40]. Plots were constructed using $\mathrm{R}$ v3.5.1 and tidyverse v1.3.0, ggpubr v0.2.4, and ggplot2 v3.2.1 libraries. Pearson's ranked correlation coefficient analysis was conducted with the R v3.5.1 corrplot v0.84 package and gene expression plots with pheatmap v1.0.12. All markdown R documents and data are available through GitHub (https://github.com/ccdmb/barley-pow-rnaseq).

To estimate Mlo exon read counts, the forward-stranded reads that mapped to the barley genome were extracted using SAMtools v1.7 view [41], with filters for SAM flags, second in pair ( $\mathrm{f} 128)$, and then the reverse reads filtered for read reverse strand $(0 \times 10)$ first in pair $(0 \times 40)(-\mathrm{f} 80)$ before being merged. Forward-stranded Mlo exons with 6-12 reads were counted for each sample using BEDTools v2.26.0 [42]. Small RNA-seq data was mapped against the barley Mlo region after normalising to $21 \mathrm{~m}$ reads per sample using the Map to Reference function in Geneious v. R8 [43]. 


\subsection{Identification of Transcription Factor Binding Sites of Highly Regulated Genes and qPCR}

One kilobase sequence regions upstream of the ATG start codon for all highly regulated genes were extracted using EnsemblPlants Biomart [28] and submitted to MEME Suite 5.1.1 [44], TOMTOM, and JASPAR CORE 2018 to identify transcription factor binding sites. Quantitative RT-PCR for the 10 most highly regulated genes containing a WRKY motif of interest was performed on a CFX96 Touch Real-Time PCR Detection System (Bio-Rad, Hercules, CA, USA) with SYBR green and Platinum DNA polymerase (Invitrogen). The barley actin gene was used as an internal reference, and relative transcript levels of biosynthesis were calculated with the $\Delta \Delta \mathrm{Ct}$ method, factoring in primer amplification efficiencies. qPCR primers used are described in Table S3 (Supplementary Materials). Student's $t$-tests between and control and inoculated were calculated for each NIL using three biological replicates with a significance value less than 0.001 .

\subsection{Gene Ontology Term Enrichment of Significantly Differentially Expressed Genes}

The top 100 genes with the largest expression variance across the five lines (Baudin (S0), S2, S12, P18, and P22) for inoculated and noninoculated samples were selected for Gene Ontology analysis. Singular enrichment analysis (SEA) of the top 100 genes was performed using agriGO [45]. The agriGO SEA selected parameter settings used were for a hypergeometric statistical test with the Yekutieli (false discovery rate under dependency) multi-testing adjustment method, significance level $\leq 0.05$, five minimum mapping entries, and Gene Ontology type Plant GO Slim [46,47]. The top 100 genes from a variance-stabilizing transformation (VST) for Baudin and Pallas NILs were also individually analysed, together with an enrichment analysis for GO terms using R topGO v2.34.0 with a classic Fisher test and significance level $\leq 0.05$.

\subsection{Chromatin Immunoprecipitation}

ChIP assays were performed as previously described $[48,49]$ with three biological replicates and three technical replicates. Antibodies used in ChIP assays included anti-H4Ac 06-866 (MilliporeSigma, St. Louis, MO, United States) anti-H3K4me3 (ab8580, Abcam PLC, Cambridge, UK), anti-H3K27me3 (Abcam, ab6002), and anti-H3K9me2 (Abcam, ab1220), and polyclonal anti-H3 histone background control (Abcam, ab1791). The amount of immunoprecipitated chromatin was determined by qPCR in different Mlo (R1-R6) regions using specific primers (Table S3, Supplementary Materials). Relative abundance of chromatin was normalised to the amount of DNA immunoprecipitated by a histone 3-specific antibody.

\subsection{Digital PCR and Bisulphite Sequencing}

Digital PCR was performed to determine the stability of the mlo-11 subunits in NILs S2 and S12 following backcrossing. Baudin (S0) was used as the wild-type control, using the protocol described in Ge et al. [9] and the primers in Table S3 (Supplementary Materials).

The EZ DNA Methylation-Direct kit (Zymo Research, Irvine, CA, USA) was used to perform bisulphite treatment of genomic DNA. First, $200 \mathrm{ng}$ of each accession with three replicates of $B g h$-infected and noninfected samples was subjected to cytosine-thiamine conversion. The converted DNA was amplified by hot-start PCR with Takara Ex Taq (Clontech Takara Cellartis, Shiga, Japan). Untreated DNA was used in control reactions. Amplified fragments were cloned into the pGEM-T vector (Promega, Madison, WI) and 18 ligated clones from each accession were Sanger sequenced.

Supplementary Materials: Supplementary materials can be found at http://www.mdpi.com/1422-0067/21/22/ 8769/s1.

Author Contributions: C.G. performed all gene silencing experiments and the RNA sequencing. L.H. and E.G.D. performed backcrosses and produced the Baudin NILs. E.W. genotyped NIL introgressions. P.M. provided bioinformatics support and analysis. W.D. advised on ChIP methodology and analysis. C.G., P.M., and S.R.E. analysed the data. C.G. conceptualised the research and drafted the original manuscript and figures with contributions from P.M. and S.R.E. All authors read and agreed to the published version of the manuscript. 
Funding: This research was funded under Grains Research and Development Corporation (GRDC) grant CUR00023 B4.

Acknowledgments: This project was supported by the Centre for Crop and Disease Management, a cooperative research vehicle of the GRDC and Curtin University. We also acknowledge the Australian Government National Collaborative Research Infrastructure Strategy (NCRIS) for providing access to Pawsey Supercomputing and Cloud resources (Nectar and Nimbus).

Conflicts of Interest: All authors declare no conflict of interest.

\section{References}

1. Kusch, S.; Panstruga, R. mlo-based resistance: An. apparently universal "weapon" to defeat powdery mildew disease. Mol. Plant-Microbe Interact. 2017, 30, 179-189. [CrossRef] [PubMed]

2. Reinstadler, A.; Müller, J.; Czembor, J.H.; Piffanelli, P.; Panstruga, R. Novel induced mlo mutant alleles in combination with site-directed mutagenesis reveal functionally important domains in the heptahelical barley Mlo protein. BMC Plant Biol. 2010, 10, 31. [CrossRef] [PubMed]

3. Piffanelli, P.; Zhou, F.; Casais, C.; Orme, J.; Jarosch, B.; Schaffrath, U.; Collins, N.C.; Panstruga, R.; Schulze-Lefert, P. The barley MLO modulator of defense and cell death is responsive to biotic and abiotic stress stimuli. Plant Physiol. 2002, 129, 1076-1085. [CrossRef] [PubMed]

4. Acevedo-Garcia, J.; Kusch, S.; Panstruga, R. Magical mystery tour: MLO proteins in plant immunity and beyond. New Phytol. 2014, 204, 273-281. [CrossRef]

5. Romero, C.C.T.; Vermeulen, J.P.; Vels, A.; Himmelbach, A.; Mascher, M.; Niks, R.E. Mapping resistance to powdery mildew in barley reveals a large-effect nonhost resistance QTL. Theor. Appl. Genet. 2018, 131, 1031-1045. [CrossRef] [PubMed]

6. Piffanelli, P.; Ramsay, L.; Waugh, R.; Benabdelmouna, A.; D’Hont, A.; Hollricher, K.; Jorgensen, J.H.; Schulze-Lefert, P.; Panstruga, R. A barley cultivation-associated polymorphism conveys resistance to powdery mildew. Nature 2004, 430, 887-891. [CrossRef] [PubMed]

7. Jørgensen, I.H. Discovery, characterization and exploitation of Mlo powdery mildew resistance in barley. Euphytica 1992, 63, 141-152. [CrossRef]

8. Dreiseitl, A. Genes for resistance to powdery mildew in European barley cultivars registered in the Czech. Republic from 2011 to 2015. Plant Breed. 2017, 136, 351-356.

9. Ge, X.; Deng, W.; Lee, Z.Z.; Lopez-Ruiz, F.J.; Schweizer, P.; Ellwood, S.R. Tempered mlo broad-spectrum resistance to barley powdery mildew in an Ethiopian landrace. Sci. Rep. 2016, 6, 29558. [CrossRef]

10. Gruner, K.; Esser, T.; Acevedo-Garcia, J.; Freh, M.; Habig, M.; Strugala, R.; Stukenbrock, E.; Schaffrath, U.; Panstruga, R. Evidence for allele-specific levels of enhanced susceptibility of wheat mlo mutants to the hemibiotrophic fungal pathogen Magnaporthe oryzae pv. Triticum. Genes 2020, 11, 517. [CrossRef]

11. Zhao, T.; Zhan, Z.; Jiang, D. Histone modifications and their regulatory roles in plant development and environmental memory. J. Genet. Genom. 2019, 46, 467-476. [CrossRef] [PubMed]

12. Law, J.A.; Jacobsen, S.E. Establishing, maintaining and modifying DNA methylation patterns in plants and animals. Nat. Rev. Genet. 2010, 11, 204-220. [CrossRef] [PubMed]

13. Matzke, M.; Kanno, T.; Daxinger, L.; Huettel, B.; Matzke, A.J.M. RNA-mediated chromatin-based silencing in plants. Curr. Opin. Cell Biol. 2009, 21, 367-376. [CrossRef] [PubMed]

14. Saze, H.; Shiraishi, A.; Miura, A.; Kakutani, T. Control of genic DNA methylation by a jmjC domain-containing protein in Arabidopsis thaliana. Science 2008, 319, 462. [CrossRef] [PubMed]

15. Javaid, N.; Choi, S. Acetylation- and methylation-related epigenetic proteins in the context of their targets. Genes 2017, 8, 196. [CrossRef]

16. Kuo, M.H.; Allis, C.D. Roles of histone acetyltransferases and deacetylases in gene regulation. BioEssays 1998, 20, 615-626.

17. Büschges, R.; Hollricher, K.; Panstruga, R.; Simons, G.; Wolter, M.; Frijters, A.; van Daelen, R.; van der Lee, T.; Diergaarde, P.; Groenendijk, J.; et al. The barley Mlo gene: A novel control element of plant pathogen resistance. Cell 1997, 88, 695-705. [CrossRef]

18. Humphry, M.; Bednarek, P.; Kemmerling, B.; Koh, S.; Stein, M.; Göbel, U.; Stüber, K.; Piślewska-Bednarek, M.; Loraine, A.; Schulze-Lefert, P.; et al. A regulon conserved in monocot and dicot plants defines a functional module in antifungal plant immunity. Proc. Natl. Acad. Sci. USA 2010, 107, 21896. [CrossRef] 
19. Huot, B.; Yao, J.; Montgomery, B.L.; He, S.Y. Growth-defense tradeoffs in plants: A balancing act to optimize fitness. Mol. Plant 2014, 7, 1267-1287. [CrossRef]

20. Neuser, J.; Metzen, C.C.; Dreyer, B.H.; Feulner, C.; van Dongen, J.T.; Schmidt, R.R.; Schippers, J.H.M. HBI1 mediates the trade-off between growth and immunity through its impact on apoplastic ROS homeostasis. Cell Rep. 2019, 28, 1670-1678.e3. [CrossRef]

21. Kølster, P.; Munk, L.; Stølen, O.; Løhde, J. Near-Isogenic barley lines with genes for resistance to powdery mildew. Crop Sci. 1986, 5, 903-907. [CrossRef]

22. An, Q.; Hückelhoven, R.; Kogel, K.-H.; Van Bel, A.J.E. Multivesicular bodies participate in a cell wall-associated defence response in barley leaves attacked by the pathogenic powdery mildew fungus. Cell. Microbiol. 2006, 8, 1009-1019. [CrossRef] [PubMed]

23. Cai, Q.; Qiao, L.; Wang, M.; He, B.; Lin, F.-M.; Palmquist, J.; Huang, S.-D.; Jin, H. Plants send small RNAs in extracellular vesicles to fungal pathogen to silence virulence genes. Science 2018, 360, 1126. [CrossRef] [PubMed]

24. Ding, Y.; Wang, X.; Su, L.; Zhai, J.; Cao, S.; Zhang, D.; Liu, C.; Bi, Y.; Qian, Q.; Cheng, Z.; et al. SDG714, a histone H3K9 methyltransferase, is involved in Tos17 DNA methylation and transposition in rice. Plant Cell 2007, 19, 9-22. [CrossRef] [PubMed]

25. Bailey, T.L.; Boden, M.; Buske, F.A.; Frith, M.; Grant, C.E.; Clementi, L.; Ren, J.; Li, W.W.; Noble, W.S. MEME SUITE: Tools for motif discovery and searching. Nucleic Acids Res. 2009, 37, W202-W208. [CrossRef] [PubMed]

26. Rushton, P.J.; Torres, J.T.; Parniske, M.; Wernert, P.; Hahlbrock, K.; Somssich, I.E. Interaction of elicitor-induced DNA-binding proteins with elicitor response elements in the promoters of parsley PR1 genes. EMBO J. 1996, 15, 5690-5700. [CrossRef]

27. Rushton, P.J.; Somssich, I.E.; Ringler, P.; Shen, Q.J. WRKY transcription factors. Trends Plant Sci. 2010, 15, 247-258. [CrossRef]

28. EnsemblPlants. International Barley Genome Sequencing Consortium (IBSC) v 2. Available online: https://plants.ensembl.org/Hordeum_vulgare/Info/Index (accessed on 1 August 2019).

29. Wolter, M.; Hollricher, K.; Salamini, F.; Schulze-Lefert, P. The mlo resistance alleles to powdery mildew infection in barley trigger a developmentally controlled defence mimic phenotype. Mol. Gen. Genet. 1993, 239, 122-128. [CrossRef]

30. Chowdhury, J.; Henderson, M.; Schweizer, P.; Burton, R.A.; Fincher, G.B.; Little, A. Differential accumulation of callose, arabinoxylan and cellulose in nonpenetrated versus penetrated papillae on leaves of barley infected with Blumeria graminis f. sp. hordei. New Phytol. 2014, 204, 650-660. [CrossRef]

31. Hackenberg, M.; Rueda, A.; Gustafson, P.; Langridge, P.; Shi, B.-J. Generation of different sizes and classes of small RNAs in barley is locus, chromosome and/or cultivar-dependent. BMC Genom. 2016, 17, 735. [CrossRef]

32. Axtell, M.J. Classification and comparison of small RNAs from plants. Annu. Rev. Plant Biol. 2013, 64, 137-159. [CrossRef] [PubMed]

33. O'Brien, J.A.; Daudi, A.; Butt, V.S.; Paul Bolwell, G. Reactive oxygen species and their role in plant defence and cell wall metabolism. Planta 2012, 236, 765-779. [CrossRef] [PubMed]

34. Tucker, M.A.; Jayasena, K.; Ellwood, S.R.; Oliver, R.P. Pathotype variation of barley powdery mildew in Western Australia. Australas. Plant Pathol. 2013, 42, 617-623. [CrossRef]

35. Mains, E.B.; Dietz, S.M. Physiologic forms of barley mildew, Erysiphe graminis hordei Marchal. Phytopathology 1930, 20, 229-239.

36. Andrews, S. FastQC. Available online: http://www.bioinformatics.babraham.ac.uk/projects/fastqc/ (accessed on 8 August 2020).

37. Bolger, A.M.; Lohse, M.; Usadel, B. Trimmomatic: A flexible trimmer for Illumina sequence data. Bioinformatics 2014, 30, 2114-2120.

38. Dobin, A.; Davis, C.A.; Schlesinger, F.; Drenkow, J.; Zaleski, C.; Jha, S.; Batut, P.; Chaisson, M.; Gingeras, T.R. STAR: Ultrafast universal RNA-seq aligner. Bioinformatics 2012, 29, 15-21. [CrossRef]

39. Love, M.I.; Huber, W.; Anders, S. Moderated estimation of fold change and dispersion for RNA-seq data with DESeq2. Genome Biol. 2014, 15, 550. [CrossRef]

40. Benjamini, Y.; Hochberg, J. Controlling the false discovery rate: A practical and powerful approach to multiple testing. J. R. Stat. Soc. Ser. B 1995, 57, 289-300. 
41. Li, H.; Handsaker, B.; Wysoker, A.; Fennell, T.; Ruan, J.; Homer, N.; Marth, G.; Abecasis, G.; Durbin, R. The Sequence Alignment/Map format and SAMtools. Bioinformatics 2009, 25, 2078-2079. [CrossRef]

42. Quinlan, A.R. BEDTools: The Swiss-army tool for genome feature analysis. Curr. Protoc. Bioinform. 2014, 47, 11.12.1-11.12.34. [CrossRef]

43. Kearse, M.; Moir, R.; Wilson, A.; Stones-Havas, S.; Cheung, M.; Sturrock, S.; Buxton, S.; Cooper, A.; Markowitz, S.; Duran, C.; et al. Geneious Basic: An. integrated and extendable desktop software platform for the organization and analysis of sequence data. Bioinformatics 2012, 28, 1647-1649.

44. Bailey, T.L.; Elkan, C. Fitting a mixture model by expectation maximization to discover motifs in biopolymers. Proc. Int. Conf. Intell. Syst. Mol. Biol. 1994, 2, 28-36.

45. Tian, T.; Liu, Y.; Yan, H.; You, Q.; Yi, X.; Du, Z.; Xu, W.; Su, Z. agriGO v2.0: A GO analysis toolkit for the agricultural community, 2017 update. Nucleic Acids Res. 2017, 45, W122-W129. [PubMed]

46. Ashburner, M.; Ball, C.A.; Blake, J.A.; Botstein, D.; Butler, H.; Cherry, J.M.; Davis, A.P.; Dolinski, K.; Dwight, S.S.; Eppig, J.T.; et al. Gene Ontology: Tool for the unification of biology. Nat. Genet. 2000, 25, $25-29$. [CrossRef] [PubMed]

47. The Gene Ontology Consortium. The Gene Ontology Resource: 20 years and still GOing strong. Nucleic Acids Res. 2019, 47, D330-D338. [CrossRef] [PubMed]

48. Bowler, C.; Benvenuto, G.; Laflamme, P.; Molino, D.; Probst, A.V.; Tariq, M.; Paszkowski, J. Chromatin techniques for plant cells. Plant J. 2004, 39, 776-789. [CrossRef] [PubMed]

49. Deng, W.; Buzas, D.M.; Ying, H.; Robertson, M.; Taylor, J.; Peacock, W.J.; Dennis, E.S.; Helliwell, C. Arabidopsis Polycomb Repressive Complex 2 binding sites contain putative GAGA factor binding motifs within coding regions of genes. BMC Genomics 2013, 14, 593. [CrossRef]

Publisher's Note: MDPI stays neutral with regard to jurisdictional claims in published maps and institutional affiliations. 